\title{
WEYL GROUPS, THE HARD LEFSCHETZ THEOREM, AND THE SPERNER PROPERTY*
}

\author{
RICHARD P. STANLEY $\dagger$
}

\begin{abstract}
Techniques from algebraic geometry, in particular the hard Lefschetz theorem, are used to show that certain finite partially ordered sets $Q^{X}$ derived from a class of algebraic varieties $X$ have the $k$-Sperner property for all $k$. This in effect means that there is a simple description of the cardinality of the largest subset of $Q^{X}$ containing no $(k+1)$-element chain. We analyze, in some detail, the case when $X=G / P$, where $G$ is a complex semisimple algebraic group and $P$ is a parabolic subgroup. In this case, $Q^{X}$ is defined in terms of the "Bruhat order" of the Weyl group of $G$. In particular, taking $P$ to be a certain maximal parabolic subgroup of $G=S O(2 n+1)$, we deduce the following conjecture of Erdös and Moser: Let $S$ be a set of $2 \ell+1$ distinct real numbers, and let $T_{1}, \cdots, T_{k}$ be subsets of $S$ whose element sums are all equal. Then $k$ does not exceed the middle coefficient of the polynomial $2(1+q)^{2}\left(1+q^{2}\right)^{2} \cdots\left(1+q^{\ell}\right)^{2}$, and this bound is best possible.
\end{abstract}

1. The Sperner property. Let $P$ be a finite partially ordered set (or poset, for short), and assume that every maximal chain of $P$ has length $n$. We say that $P$ is graded of rank $n$. Thus $P$ has a unique rank function $\rho: P \rightarrow\{0,1, \cdots, n\}$ satisfying $\rho(x)=0$ if $x$ is a minimal element of $P$, and $\rho(y)=\rho(x)+1$ if $y$ covers $x$ in $P$ (i.e., if $y>x$ and no $z \in P$ satisfies $y>z>x)$. If $\rho(x)=i$, then we say that $x$ has rank $i$. Define $P_{i}=\{x \in P: \rho(x)=i\}$ and set $p_{i}=p_{i}(P)=\operatorname{card} P_{i}$. The polynomial $F(P, q)=p_{0}+p_{1} q+\cdots+p_{n} q^{n}$ is called the rank-generating function of $P$. We say that $P$ is rank-symmetric if $p_{i}=p_{n-i}$ for all $i$, and that $P$ is rank-unimodal if $p_{0} \leqq p_{1} \leqq \cdots \leqq p_{i} \geqq p_{i+1} \geqq \cdots \geqq p_{n}$ for some $i$.

An antichain (also called a Sperner family or clutter) is a subset $A$ of $P$, such that no two distinct elements of $A$ are comparable. The poset $P$ is said to have the Sperner property (or property $\mathrm{S}_{1}$ ) if the largest size of an antichain is equal to $\max \left\{p_{i}: 0 \leqq i \leqq n\right\}$. More generally, if $k$ is a positive integer then $P$ is said to have the $k$-Sperner property (or property $\left.\mathrm{S}_{k}\right)$ if the largest subset of $P$ containing no $(k+1)$-element chain has cardinality $\max \left\{p_{i_{1}}+\cdots+p_{i_{k}}: 0 \leqq i_{1}<\cdots<i_{k} \leqq n\right\}$. If $P$ has property $\mathrm{S}_{k}$ for all $k \leqq n$, then following [21] we say that $P$ has property $S$. For further information concerning the Sperner property and related concepts, see for instance [15], [16], [17].

Using some results from algebraic geometry, we will give several new classes of graded posets which have property $S$. These posets will all be rank-symmetric and rank-unimodal. First we must consider a property of posets related to property $S$. Suppose $P$ is graded of rank $n$ and is rank-symmetric. Again following [21], we say that $P$ has property $\mathrm{T}$ if for all $0 \leqq i \leqq[n / 2]$, there exist $p_{i}$ pairwise disjoint saturated chains $x_{i}<x_{i+1}<\cdots<x_{n-i}$ where $x_{j} \in P_{j}$. It is clear that $P$ is then rank-unimodal.

LEMMA 1.1. Let $P$ be a finite graded rank-symmetric poset of rank $n$. The following three conditions are equivalent:

(i) $P$ is rank-unimodal and has property $\mathrm{S}$.

(ii) $P$ has property $\mathrm{T}$.

(iii) Let $V_{i}$ be the complex vector space with basis $P_{i}$. Then for $0 \leqq i<n$, there exist linear transformations $\varphi_{i}: V_{i} \rightarrow V_{i+1}$ satisfying the following two properties:

(a) If $0 \leqq i \leqq[n / 2]$, then the composite transformation $\varphi_{n-i-1} \varphi_{n-i-2} \cdots$ $\varphi_{i+1} \varphi_{i}: V_{i} \rightarrow V_{n-i}$ is invertible.

(b) Let $x \in P_{i}$ and $\varphi_{i}(x)=\sum_{y \in P_{i+1}} c_{y} y$. Then $c_{y}=0$ unless $x<y$.

Proof. (i) $\Leftrightarrow$ (ii). This is a special case of [21, Thms. 2 and 3$]$.

* Received by the editors June 1, 1979.

$\dagger$ Department of Mathematics, Massachusetts Institute of Technology, Cambridge, Massachusetts 02139. The research was supported in part by the National Science Foundation under Grant MCS 77-01947. 
(iii) $\Rightarrow$ (ii). (I am grateful to Joseph Kung for supplying the following argument, which is considerably simpler than my original proof.) Assume (iii). Identify $\varphi_{i}$ with its matrix with respect to the bases $P_{i}$ and $P_{i+1}$. If $\varphi$ is a matrix whose rows are indexed by a set $S$ and whose columns are indexed by $T$, and if $S^{\prime} \subset S$ and $T^{\prime} \subset T$, then let $\varphi\left[S^{\prime}, T^{\prime}\right]$ denote the submatrix of $\varphi$ with rows indexed by $S^{\prime}$ and columns by $T^{\prime}$. By the Binet-Cauchy theorem (e.g., $[1, \S 36]$ ) we have

$$
\begin{aligned}
\operatorname{det}\left(\varphi_{n-i-1} \cdots \varphi_{i}\right)= & \sum\left(\operatorname{det} \varphi_{i}\left[Q_{i}, Q_{i+1}\right]\right) \\
& \cdot\left(\operatorname{det} \varphi_{i+1}\left[Q_{i+1}, Q_{i+2}\right]\right) \cdots\left(\operatorname{det} \varphi_{n-i-1}\left[Q_{n-i-1}\left[Q_{n-i}\right]\right),\right.
\end{aligned}
$$

where the sum is over all sequences of subsets $Q_{i}=P_{i}, Q_{i+1} \subset P_{i+1}$, $Q_{i+2} \subset P_{i+2}, \cdots, Q_{n-i-1} \subset P_{n-i-1}, Q_{n-i}=P_{n-i}$ such that $\left|Q_{i+1}\right|=\left|Q_{i+2}\right| \cdots=\left|Q_{n-i-1}\right|=$ $p_{i}$. By (a), some term in the above sum is nonzero. Hence, the expansion of each factor $\operatorname{det} \varphi_{k}\left[Q_{k}, Q_{k+1}\right]$ in this term contains a nonzero term. By (b), this nonzero term defines a map $\sigma: Q_{k} \rightarrow Q_{k+1}$ such that $x<\sigma(x)$ for all $x \in Q_{k}$. Piecing together these twoelement chains over all $k$ yields (ii).

(ii) $\Rightarrow$ (iii). The steps of the above argument can be reversed, provided we pick the $\varphi_{i}$ 's as generically as possible, i.e., all the entries of the matrices $\varphi_{0}, \varphi_{1}, \cdots, \varphi_{n-1}$ should be chosen to be algebraically independent over $\mathbb{Q}$, except for entries forced to equal 0 by condition (b). This completes the proof.

2. Varieties with cellular decompositions. We now are in a position to invoke algebraic geometry. Let $X$ be a complex projective variety of complex dimension $n$. Suppose that there are finitely many pairwise-disjoint subsets $C_{i}$ of $X$, each isomorphic as an algebraic variety to complex affine space of some dimension $n_{i}$, such that (i) the union of the $C_{i}$ 's is $X$, and (ii) $\bar{C}_{i}-C_{i}$ is a union of some of the $C_{j}$ 's. (Here $\bar{C}_{i}$ denotes the closure of $C_{i}$ either in the Hausdorff or Zariski topology-under the present circumstances the two closures coincide.) Following [4, p. 500], we then say that the $C_{i}$ 's form a cellular decomposition of $X$. The simplest and most familiar example is complex projective space $\mathbb{P}^{n}$ itself. Recall that $\mathbb{P}^{n}$ may be regarded as the set of nonzero $(n+1)$-tuples $x=\left(x_{0}, x_{1}, \cdots, x_{n}\right) \in \mathbb{C}^{n+1}$, modulo the equivalence relation $x \sim$ $\lambda x\left(\lambda \in \mathbb{C}^{*}\right)$. The set of elements of $\mathbb{P}^{n}$ of the form $\left(0, \cdots, 0,1, x_{n-i+1}, \cdots, x_{n}\right)$ forms a subvariety isomorphic to $\mathbb{C}^{i}$. Hence we have the cellular decomposition $\mathbb{P}^{n}=\mathbb{C}^{n} \cup \mathbb{C}^{n-1} \cup \cdots \cup \mathbb{C}^{0}$

If $X$ is any complex projective variety and $Y$ is a closed subvariety, then e.g., by [4] or [18, Chap. 5, § 4], $Y$ represents an element (cocycle) [Y] of the cohomology group $H^{*}(X, \mathbb{C})$. If $X$ is irreducible of (complex) dimension $n$, and $Y$ is irreducible of dimension $m$, then in fact $[Y] \in H^{2(n-m)}(X, \mathbb{C})$. If $X$ is irreducible of dimension $n$ and has a cellular decomposition $\left\{C_{i}\right\}$, it follows that the closures $\bar{C}_{i}$ represent cohomology classes $\left[\bar{C}_{i}\right] \in H^{2(n-m)}(X, \mathbb{C})$ where $C_{i} \cong \mathbb{C}^{m}$. (For this fact, we don't need condition (ii) in our definition of cellular decomposition.) The following fundamental result concerning varieties with a cellular decomposition appears in $[4$, p. 501], $[22, \S 6]$ in the case when $X$ is nonsingular. The extension to singular varieties follows from [14]. (Again, condition (ii) is not actually necessary.)

THEOREM 2.1. Let $X$ be a complex projective variety of complex dimension $n$, and suppose that $X$ has a cellular deomposition $\left\{C_{i}\right\}$. Then the cohomology classes $\left[\bar{C}_{i}\right]$ form a basis (over $\mathbb{C}$ ) for $H^{*}(X, \mathbb{C})$. In particular, $H^{2 m+1}(X, \mathbb{C})=0$ for all $m \in \mathbb{Z}$, while if $X$ is irreducible then $H^{2(n-m)}(X, \mathbb{C})$ has a basis consisting of those classes $\left[\bar{C}_{i}\right]$ for which $C_{i} \cong \mathbb{C}^{m}$.

Now given a cellular decomposition $\left\{C_{i}\right\}$ of $X$, define a partial ordering $Q^{X}=$ $Q^{X}\left(C_{1}, C_{2}, \cdots\right)$ on the $C_{i}$ 's by setting $C_{i} \geqq C_{j}$ in $Q^{X}$ if $C_{i} \subset \bar{C}_{i}$. If $X$ is irreducible of 
dimension $n$, then it can be shown, using standard techniques from algebraic geometry, that $Q^{X}$ is graded of rank $n$, with the rank function given by $\rho(C)=n-\operatorname{dim} C$. If, moreover, $X$ is nonsingular, then Poincaré duality implies that $Q^{X}$ is rank-symmetric. Theorem 2.1 then implies that we may identify the vector space $V_{i}$ of Lemma 1.1 (iii) with $H^{2 i}(X, \mathbb{C})$ by identifying $C \in Q_{i}^{X}$ with $[\bar{C}] \in H^{2 i}(X, \mathbb{C})$.

We now wish to define linear transformations $\varphi_{i}: V_{i} \rightarrow V_{i+1}$ (or equivalently, $\varphi_{i}: H^{2 i}(X, \mathbb{C}) \rightarrow H^{2(i+1)}(X, \mathbb{C})$ ) satisfying conditions (a) and (b) of Lemma 1.1 (iii). This will enable us to conclude that $Q^{X}$ has property $S$. Let $Y$ be a hyperplane section of $X$, i.e., the intersection of $X$ (regarded as being imbedded in some projective space $\mathbb{P}^{N}$ ) with a hyperplane of $\mathbb{P}^{N}$. If $X$ is irreducible, then $Y$ is a closed subvariety of $X$ of dimension $n-1$ which represents a cohomology class $[Y] \in H^{2}(X, \mathbb{C})$. The cup product operation on cohomology then yields a linear transformation $\varphi_{i}: H^{2 i}(X, \mathbb{C}) \rightarrow$ $H^{2(i+1)}(X, \mathbb{C})$ defined as multiplication by $[Y]$. In other words, $\varphi_{i}(K)=[Y] \cdot K$. We now verify that when $X$ is nonsingular and irreducible (so $Q^{X}$ is graded and ranksymmetric), then these linear transformations $\varphi_{i}$ satisfy conditions (a) and (b) of Lemma 1.1 (iii). First we dispose of condition (b). I am grateful to Steve Kleiman for providing a proof of this result.

LEMMA 2.2. Let $X$ be a complex projective variety with a cellular decomposition $\left\{C_{i}\right\}$, and let $Y$ be a hyperplane section (or in fact any closed subvariety) of X. If $[Y] \cdot\left[\bar{C}_{i}\right]=$ $\sum \alpha_{j}\left[\bar{C}_{j}\right]$ in $H^{*}(X, \mathbb{C})$, then $\alpha_{j}=0$ unless $C_{j} \subset \bar{C}_{i}$.

Proof. Let $A(W)$ denote the Chow group of the variety $W$, i.e., the group of cycles modulo rational equivalence. If $W$ is nonsingular and has a cellular decomposition $\left\{D_{i}\right\}$, then it is mentioned in $[22, \S 6]$ that the cycles $\bar{D}_{i}$ form a basis for $A(W)$, and that the corresponding map $A(W) \rightarrow H^{*}(W, \mathbb{Z})$ is an isomorphism of groups. It follows from [14] that this result continues to hold when $W$ is singular. Now returning to our hypotheses, the $C_{j}$ 's contained in $\bar{C}_{i}$ form a cellular decomposition of $\bar{C}_{i}$. Hence a hyperplane section of $\bar{C}_{i}$ is rationally equivalent to a linear combination of the $\bar{C}_{j}$ that are contained in $\bar{C}_{i}$. A priori, the rational equivalence is on $\bar{C}_{i}$, but it may be considered as a rational equivalence on $X$. Hence $\alpha_{j}=0$ unless $C_{j} \subset \bar{C}_{i}$ because the $\left[\overline{\mathrm{C}}_{\mathrm{i}}\right]$ are linearly independent in $H^{*}(X, \mathbb{C})$.

Lemma 2.2 shows that condition (b) of Lemma 1.1 (iii) holds for $Q^{X}$ (assuming $X$ is nonsingular and irreducible, so we know $Q^{X}$ is graded and rank-symmetric). Condition (a) is implied by the following basic result, known as the "hard Lefschetz theorem" (although the first rigorous proof was given by Hodge). See [34] for a brief history and survey of this theorem, and for its extension to characteristic $p$. Other references include [24, p. 187], [29], [10, Corollary, p. 75], [30, p. 44], [19, Chap. 0, § 7].

LEMMA 2.3 (the hard Lefschetz theorem). Let $X$ be a nonsingular irreducible complex projective variety of complex dimension $n$. Let $Y$ be a hyperplane section of $X$. If $0 \leqq i \leqq n$, then the linear transformation $H^{i}(X, \mathbb{C}) \rightarrow H^{2 n-i}(X, \mathbb{C})$ given by multiplication by $[Y]^{n-i}$ is an isomorphism.

Putting Lemmas 1.1, 2.2, and 2.3 together, we obtain the main result of this paper.

THEOREM 2.4. Let $X$ be a nonsingular irreducible complex projective variety of complex dimension $n$ with a cellular decomposition $\left\{C_{i}\right\}$. Then $Q^{X}$ is graded of rank $n$, rank-symmetric, rank-unimodal, and has property $\mathrm{S}$.

For future use, we record the following simple result. The proof is evident.

Proposition 2.5. Let $X$ and $Y$ be complex projective varieties, with cellular decompositions $\left\{C_{i}\right\}$ and $\left\{D_{j}\right\}$ respectively. Then the product variety $X \times Y$ has a cellular decomposition with cells $C_{i} \times D_{i}$, and $Q^{X \times Y} \cong Q^{X} \times Q^{Y}$.

It follows from Theorem 2.4 and Proposition 2.5 that if $P=Q^{X}$ and $P^{\prime}=Q^{Y}$ for nonsingular irreducible complex projective varieties $X$ and $Y$, each having a cellular 
decomposition, then $P \times P^{\prime}$ has property S. More generally, Canfield [7] and independently Proctor, Saks, and Sturtevant [36] have shown that the product $P \times P^{\prime}$ of any two graded, rank-symmetric, rank-unimodal posets $P$ and $P^{\prime}$, each with property $\mathrm{S}$, also has property S. (An even more general result has subsequently been proved by Saks [37].) For our purposes, however, it suffices to consider only Proposition 2.5.

3. Weyl groups. It remains to find interesting examples of varieties $X$ with cellular decompositions and to describe the resulting posets $Q^{X}$. The best known examples of such varieties are the following. Let $G$ be a complex semisimple algebraic group, and let $P$ be a parabolic subgroup of $G$ (i.e., a closed subgroup which contains a maximal solvable subgroup $B$ of $G$. $B$ is known as a Borel subgroup.) Then the coset space $G / P$ has the structure of a non-singular irreducible complex projective variety, and the Bruhat decomposition of $G$ affords a cellular decomposition $\left\{C_{i}\right\}$ of $G / P$. The cells $C_{i}$ are known as generalized Schubert cells. See [5, § 3] for further details.

When $X=G / P$, a description of the poset $Q^{X}$ can be given in terms of the Weyl groups $W$ of $G$, and $W_{J}$ of $P[5, \S 3],[11]$ as follows. Every Weyl group $W$ is a finite Coxeter group, i.e., $W$ is a finite group with a finite set $S=\left\{s_{1}, \ldots, s_{m}\right\}$ of generators such that for all $1 \leqq k \leqq m, 1 \leqq i<j \leqq m$ and certain integers $n_{i j} \geqq 2, W$ is defined by the relations $s_{k}^{2}=1$ and $\left(s_{i} s_{j}\right)^{n_{i j}}=1$. The pair $(W, S)$ is called a Coxeter system.

A parabolic subgroup of $W$ (with respect to $S$ ) is any subgroup $W_{J}$ generated by a subset $J$ of $S$. Thus $W_{\phi}=\{1\}$ and $W_{S}=W$. The length $\ell(w)$ of an element $w \in W$ is the smallest integer $q \geqq 0$ for which $w$ is a product of $q$ elements of $S$. Define a partial order, called the Bruhat order, on $W$ as follows. We say $w \leqq w^{\prime}$ if there exist conjugates $t_{1}, \cdots, t_{j}$ of the elements of $S$ such that $w^{\prime}=w t_{1} t_{2} \cdots t_{j}$ and $\ell\left(w t_{1} t_{2} \cdots t_{i+1}\right)>$ $\ell\left(w t_{1} t_{2} \cdots t_{i}\right)$ for all $0 \leqq i<j$. The following properties (among others) of the Bruhat order of a finite Coxeter group $W$ are known:

1. The Bruhat order makes $W$ into a graded poset (which we still call $W$ ).

2. The function $\ell$ is the rank function of $W$, and the rank-generating function of $W$ is given by

$$
F(W, q)=\prod_{i=1}^{m}\left(1+q+q^{2}+\cdots+q^{e_{i}}\right)
$$

for certain positive integers $e_{i}$ known as the exponents of $W$. One may regard (1) as the definition of the exponents. For other equivalent definitions, see, e.g., [6, Chap. $5, \S 6.2]$ or [8, Chap. 10]. Note that (1) implies the well-known fact that $|W|=\prod\left(e_{i}+1\right)$, and that $W$ has rank $e_{1}+\cdots+e_{m}$.

3. If $J \subset S$, then each coset $w W_{J}$ of $W_{J}$ in $W$ contains a unique element $w_{J}$ of minimal length. For any $v \in W_{J}$ we have $\ell\left(w_{J} v\right)=\ell\left(w_{J}\right)+\ell(v)$.

4. Let $W^{J}$ be the set of minimal length coset representatives $w_{J}$. Then $W^{J}$ is a graded subposet of $W$ such that the rank function of $W^{J}$ is the restriction of the rank function of $W$.

5. $\left(W_{J}, J\right)$ is itself a finite Coxeter system, say with exponents $f_{1}, \cdots, f_{t}$. Then $W^{J}$ has the rank-generating function

$$
F\left(W^{J}, q\right)=\frac{F(W, q)}{F\left(W_{J}, q\right)}=\frac{\prod_{i=1}^{m}\left(1+q+q^{2}+\cdots+q^{e_{i}}\right)}{\prod_{j=1}^{t}\left(1+q+q^{2}+\cdots+q^{f_{i}}\right)} .
$$

For proofs of these results and further information on Coxeter groups, see e.g., [6], [8], [11]. For a connection between the posets $W^{J}$ and combinatorics, different from the one given here, see [23].

Now we return to the varieties $X=G / P$, where $G$ is a complex semisimple algebraic group and $P$ a parabolic subgroup of $G$. It is known [6, p. 29], [5, § 3] that the parabolic subgroups of $G$ containing a given Borel subgroup $B$ are in one-to-one 
correspondence with the parabolic subgroups $W_{J}$ of the Weyl group $W$ of $G$ (with respect to a fixed set $S$ of Coxeter generators of $W$ ). Moreover, the poset $Q^{X}$ corresponding to the cellular decomposition of $X=G / P$ obtained from the Bruhat decomposition of $G$ is isomorphic to the partial order on $W^{J}$ defined above. Hence from Theorem 2.4 we conclude:

THEOREM 3.1. Let $(W, S)$ be a Coxeter system for which $W$ is a Weyl group. Let $J \subset S$ and let $W^{J}$ be the poset defined above. Then $W^{J}$ is rank-symmetric, rank-unimodal, and has property $\mathrm{S}$.

A Coxeter system $(W, S)$ is irreducible if one cannot write $S$ as a nontrivial disjoint union $T \cup T^{\prime}$ such that $W=W_{T} \times W_{T^{\prime}}$. If $(W, S)$ is reducible, say $W=W_{T} \times W_{T^{\prime}}$, then we also have $W=W_{T} \times W_{T^{\prime}}$ as posets, and similarly for $W^{J}$. Thus by Proposition 2.5 nothing is lost by considering only irreducible Coxeter systems. Now all finite irreducible Coxeter systems are known (e.g., [6, p. 193]). There are the infinite families of type $A_{n}(n \geqq 1), B_{n}(n \geqq 2)$, and $D_{n}(n \geqq 4)$, together with seven "exceptional" systems $E_{6}, E_{7}$, $E_{8}, F_{4}, G_{2}, H_{3}, H_{4}$ and the dihedral groups $I_{2}(p)$ of order $2 p$ for $p=5$ or $p \geqq 7 .\left(I_{2}(3)\right.$ coincides with $A_{2}, I_{2}(4)$ with $B_{2}$, and $I_{2}(6)$ with $G_{2}$.) For all of these systems $(W, S), W$ is a Weyl group except for $H_{3}, H_{4}, I_{2}(p), p=5$ or $p \geqq 7$. It is easy to check that Theorem 3.1 remains valid for the dihedral groups $I_{2}(p)$, and for $H_{3}$. Presumably the remaining case $H_{4}$ can also be checked directly, so in fact one could determine those finite Coxeter systems (probably all of them) for which Theorem 3.1 remains valid.

4. Type $\boldsymbol{A}_{n}$. We now want to describe the posets $W^{J}$ in greater detail, for the types $A_{n}, B_{n}, D_{n}$. First consider $A_{n-1}$. Then $W$ is the symmetric group $\widetilde{\subseteq}_{n}$ of all permutations of $\{1,2, \cdots, n\}$. The exponents are $1,2, \cdots, n-1$, and as Coxeter generators we may take the "adjacent transpositions" $s_{i}=(i, i+1), 1 \leqq i \leqq n-1$. Regard a permutation $\pi \in \widetilde{S}_{n}$ as a linear array $a_{1} a_{2} \cdots a_{n}$, where $\pi(i)=a_{i}$. Then a direct translation of the definition of the Bruhat order yields the following: $\pi \leqq \sigma$ in $W$ if $\sigma$ can be obtained from $\pi$ by a sequence of operations which interchange $i$ and $j$ in a permutation $a_{1} a_{2} \cdots a_{n}$ provided $i$ appears to the left of $j$ and $i<j$. We abbreviate this operation as

$$
i<j \longrightarrow j>i \text {. }
$$

Thus the notation " $i<j$ " in (3) means that $i$ and $j$ appear in the given order (i.e., $i$ to the left of $j$ ) and $i<j$. For instance, $213 \leqq 312$ (obtained by $2<3 \longrightarrow 3>2$ ) and $24153 \leqq$ 35241 (obtained, e.g., by $2<3 \rightarrow 3<2,1<2 \rightarrow 2>1,4<5 \rightarrow 5>4$ ). The rank $\ell(\pi)$ of $\pi=a_{1} a_{2} \cdots a_{n} \in W$ is equal to the number $i(\pi)$ of inversions of $\pi$, i.e., the number of pairs $(i, j)$ for which $i<j$ and $a_{i}>a_{j}$. Thus $12 \cdots n$ is the unique permutation of rank 0 and $n \cdots 21$ is the unique permutation of highest rank $\left(\begin{array}{l}n \\ 2\end{array}\right)$. It is well-known (e.g., [9, $\S 6.4])$ that

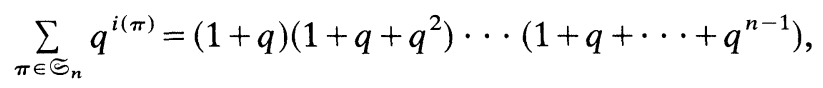

which of course agrees with (1). Figure 1 depicts the Bruhat order of $\Im_{3}$.

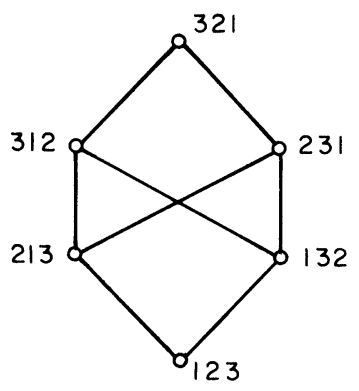

FIG. 1 
Now let $J \subset S=\left\{s_{1}, \cdots, s_{n-1}\right\}$ where $s_{i}=(i, i+1)$. If we let $\subseteq(a, b)$ denote the group of all permutations of $\{a, a+1, \cdots, b\}$, then it is clear that $W_{J}=$

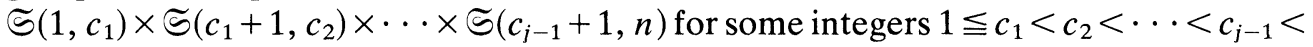
$n$, where $j=n-|J|$. If $\pi=a_{1} a_{2} \cdots a_{n} \in W$, then the coset $\pi W_{J}$ consists of all $c_{1} !\left(c_{2}-\right.$ $\left.c_{1}\right)$ ! $\cdots\left(n-c_{j-1}\right)$ ! permutations obtained from $\pi$ by permuting among themselves the elements within the sets $N_{1}=\left\{1,2, \cdots, c_{1}\right\}, N_{2}=\left\{c_{1}+1, \cdots, c_{2}\right\}, \cdots, N_{j}=$ $\left\{c_{j-1}+1, \cdots, n\right\}$. The coset representative $\pi_{J} \in \pi W_{J}$ with the least number of inversions is that element of $\pi W_{J}$ for which the elements of the above sets $N_{i}$ appear in their natural order. Hence $W^{J}$ consists of those $n ! / c_{1} !\left(c_{2}-c_{1}\right) ! \cdots\left(n-c_{j-1}\right) !$ permutations for which the elements of each of the sets $N_{i}$ appear in their natural order; or, as it is sometimes called, the set of shuffles of $N_{1}, \cdots, N_{j}$. The rank-generating function of $W^{J}$ is given by

$$
F\left(W^{J}, q\right)=\frac{(\mathbf{n}) !}{\left(\mathbf{c}_{1}\right) !\left(\mathbf{c}_{2}-\mathbf{c}_{1}\right) ! \cdots\left(\mathbf{n}-\mathbf{c}_{j-1}\right) !}
$$

where $(\mathbf{k}) !=(1-q)\left(1-q^{2}\right) \cdots\left(1-q^{k}\right)$. The right-hand side of (4) is known as a $q$-multinomial coefficient and is commonly denoted $\left[\begin{array}{c}n \\ c_{1}, c_{2}-c_{1}, \cdots, n-c_{j-1}\end{array}\right]$. Figure 2 illustrates the poset $W^{J}$ in the case $n=4, J=\{(12)\}$.

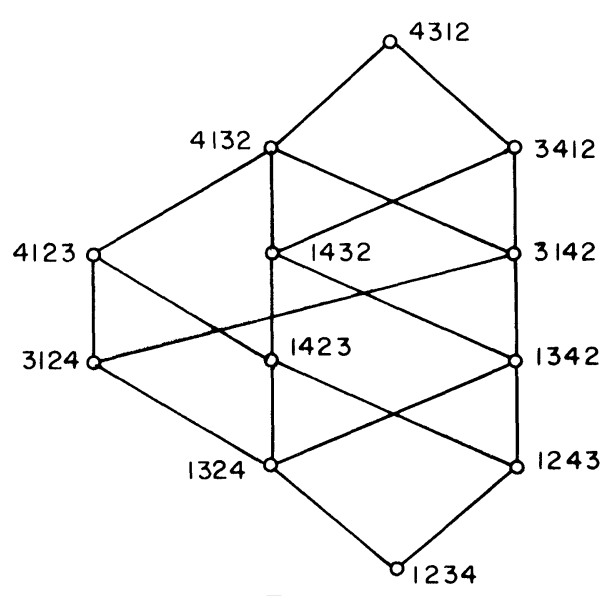

FIG. 2

If we take $W_{J}$ to be a maximal parabolic subgroup above, i.e., $|J|=n-2$, then the poset $W^{J}$ has an interesting alternative description. Suppose $J=S-\{(n-k, n-k+1)\}$, so $N_{1}=\{1,2, \cdots, n-k\}$ and $N_{2}=\{n-k+1, \cdots, n\}$. If $\pi=a_{1} a_{2} \cdots a_{n} \in W^{J}$ and $1 \leqq i \leqq k$, then set

$$
\ell_{i}(\pi)=\operatorname{card}\{j: j \text { appears to the right of } n-i+1 \text { and } j<n-i+1\} .
$$

Clearly $\ell(\pi)=\sum_{i=1}^{k} \ell_{i}(\pi)$. The mapping $\pi \mapsto\left(\ell_{1}(\pi), \cdots, \ell_{k}(\pi)\right)$ is a bijection between $W^{J}$ and all integer sequences $0 \leqq \ell_{1} \leqq \cdots \leqq \ell_{k} \leqq n-k$. Moreover, $\pi \leqq \pi^{\prime}$ in $W^{J}$ if and only if $\ell_{i} \leqq \ell_{i}^{\prime}$ for $1 \leqq i \leqq k$. Hence, $W^{J}$ is isomorphic to the poset of all partitions of integers into at most $k$ parts, with largest part at most $n-k$, i.e., a partition whose Ferrers diagram (e.g., $[9, \S 2.4])$ fits into a $k \times(n-k)$ rectangle. These partitions are ordered by inclusion of their Ferrers diagrams. Since the union and intersection of Ferrers diagrams is again a Ferrers diagram, it follows that the poset $W^{J}$ is actually a distributive lattice, which we will denote by $L(k, n-k)$. Figure 3 depicts $L(2,3)$. 


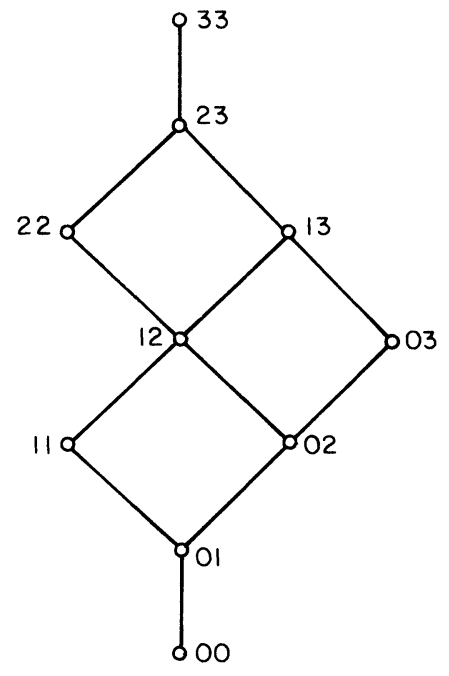

FIG. 3

In terms of the characterization [3, Thm. 3, p. 46] of a finite distributive lattice $L$ as the lattice $\mathbf{2}^{P}$ of semi-ideals (also called "order ideals" or "decreasing subsets") of a poset $P$, we have $L(k, n-k)=\mathbf{2}^{\mathbf{k} \times(\mathbf{n}-\mathbf{k})}$, where $\mathbf{i}$ denotes an $i$-element chain. The rank-generating function of this lattice is the $q$-binomial coefficient $\left[\begin{array}{l}n \\ k\end{array}\right]=$ (n)!/(k)!(n-k)!. It is by no means a priori obvious that $W^{J}$ is rank-unimodal; this was first shown essentially by Sylvester in 1878 (see [40] for historical details) and no combinatorial proof is known. I am grateful to Tony Iarrobino for originally calling to my attention that the hard Lefschetz theorem implies the unimodality of the coefficients of $\left[\begin{array}{l}n \\ k\end{array}\right]$. It was my attempt to understand this fact which eventually led to the present paper.

By applying Theorem 3.1 to the lattice $L(k, m)$, we can deduce a "multiset analogue" to a conjecture of Erdös and Moser [13, (12)]. (Regarding their actual conjecture, see Corollary 5.3 below.) I am grateful to Ranee Gupta for her comments on this result.

Corollary 4.1. Fix positive integers $k, m$, and $j$. Let $A=\left\{a_{0}, a_{1}, \cdots, a_{m}\right\}$ be a set of $m+1$ distinct real numbers. Let $B_{1}, \cdots, B_{r}$ be subsets of $A$ with exactly $k$ elements with repeated elements allowed. (One may think of $B_{s}$ as being an $m+1$-tuple $\left(\alpha_{0}, \cdots, \alpha_{m}\right.$ ) of nonnegative integers such that $\sum \alpha_{i}=k$, where $\alpha_{i}$ is the number of repetitions of $a_{i}$.) Let $\sum B_{s}$ denote the sum of the elements of $B_{s}$, i.e., $\sum B_{s}=\sum \alpha_{i} a_{i}$. Suppose that there are at most $j$ distinct numbers among $\sum B_{1}, \cdots, \sum B_{r}$. Then $r$ is less than or equal to the sum of the $j$ middle coefficients of the polynomial $\left[\begin{array}{c}m+k \\ k\end{array}\right]$. Moreover, this value of $r$ is achieved by taking $A=\{0,1, \cdots, m\}$ and $B_{1}, \cdots, B_{r}$ to have element sums consisting of the $j$ middle elements of the set $\{0,1, \cdots, k m\}$. (If $k m-j$ is even, then there are two equivalent choices of the " $j$ middle coefficients" and " $j$ middle elements.")

Proof. Regarding $B_{s}=\left(\alpha_{0}, \cdots, \alpha_{m}\right)$ associate with $B_{s}$ the sequence $\lambda_{s}=$ $\left(\ell_{1}, \cdots, \ell_{k}\right) \in L(k, m)$ defined by setting exactly $\alpha_{i}$ of the $\ell_{k}$ 's equal to $i$. It is easy to see that the subset $\left\{\lambda_{1}, \cdots, \lambda_{r}\right\}$ of $L(k, m)$ contains no $(j+1)$-element chain provided there are only $j$ distinct numbers among $\sum B_{1}, \cdots, \sum B_{r}$. The proof now follows from Theorem 3.1 and the fact that the rank-generating function of $L(k, m)$ is $\left[\begin{array}{c}k+m \\ k\end{array}\right]$. 
As a variation of the preceding corollary, we have

COROLLARY 4.2. Fix positive integers $k, m$, and $j$. Let $A^{\prime}=\left\{a_{1}, \cdots, a_{m}\right\}$ be a set of $m$ distinct nonzero real numbers. Let $B_{1}, \cdots, B_{r}$ be subsets of $A^{\prime}$ with at most $k$ elements with repeated elements allowed. Suppose that there are at most $j$ distinct numbers among $\sum B_{1}, \cdots, \sum B_{r}$. Then $r$ is less than or equal to the sum of the $j$ middle coefficients of the polynomial $\left[\begin{array}{c}m+k \\ k\end{array}\right]$. Moroever, this value of $r$ is achieved by taking $A^{\prime}=\{1, \cdots, m\}$ and $B_{1}, \cdots, B_{r}$ to have element sums consisting of the $j$ middle elements of the set $\{0,1, \cdots, k m\}$.

Proof. Apply Corollary 4.1 to the set $A=A^{\prime} \cup\{0\}$.

Remark. The cellular decomposition of $G / P$ in the case $W(G)=\Im_{n}$ and $W(P)=$ $\widetilde{S}_{k} \times \widetilde{S}_{n-k}$ can be described quite concretely. The group $G$ is given by $S L(n, \mathbb{C})$, which acts linearly on $n$-dimensional complex projective space $\mathbb{P}^{n-1}$. Let $V$ be a $(k-1)$ dimensional subspace (or $(k-1)$-plane) of $\mathbb{P}^{n-1}$, and let $P$ be the subgroup of $G$ leaving $V$ invariant. (Then $P$ is a maximal parabolic subgroup of $G$.) The coset $\phi P$ transforms $V$ into the subspace $\phi V$, and this sets up a one-to-one correspondence between $X=G / P$ and the $(k-1)$-planes in $\mathbb{P}^{n-1}$. Hence $X$ is the Grassmann manifold $G(k-1, n-1)$ of all $(k-1)$-planes in $\mathbb{P}^{n-1}$. Regard the elements of $\mathbb{P}^{n-1}$ as (equivalence classes of) $n$-tuples $\left(x_{1}, \cdots, x_{n}\right) \in \mathbb{C}^{n}-\{0\}$. A $(k-1)$-plane $V$ in $\mathbb{P}^{n-1}$ has a unique ordered basis $w_{1}, \cdots, w_{k}$ for which the matrix $\left[\begin{array}{c}w_{1} \\ \vdots \\ w_{k}\end{array}\right]$ is in row-reduced echelon form. Choose integers $0 \leqq a_{1} \leqq a_{2} \leqq \cdots \leqq a_{k} \leqq n-k$, and suppose we specify that for each $i$, the first 1 in $w_{i}$ occurs in coordinate $a_{i}+i$. The set of all such $V$ forms a subset $C\left(a_{1}, \cdots, a_{k}\right)$ of $G(k-1, n-1)$ isomorphic to $\mathbb{C}^{k(n-k)-a_{1}-\cdots-a_{k}}$; indeed, there are $n-k-a_{i}$ coordinates in $w_{i}$ which can be specified arbitrarily, and the remaining coordinates are predetermined. By considering all sequences $0 \leqq a_{1} \leqq \cdots \leqq a_{k} \leqq n-k$, we obtain a cellular decomposition of $G(k-1, n-1)$. Thus the cells $C\left(a_{1}, \cdots, a_{k}\right)$ are in one-to-one correspondence with the elements $\left(a_{1}, \cdots, a_{k}\right)$ of $L(k, n-k)$. For instance, when $k=2$ and $n=4$ the cells correspond to the following row-reduced echelon matrices:

$$
\begin{aligned}
& {\left[\begin{array}{llll}
1 & 0 & * & * \\
0 & 1 & * & *
\end{array}\right],\left[\begin{array}{llll}
1 & * & 0 & * \\
0 & 0 & 1 & *
\end{array}\right],\left[\begin{array}{llll}
1 & * & * & 0 \\
0 & 0 & 0 & 1
\end{array}\right]} \\
& C(0,0) \quad C(0,1) \quad C(0,2) \\
& {\left[\begin{array}{llll}
0 & 1 & 0 & * \\
0 & 0 & 1 & *
\end{array}\right],\left[\begin{array}{llll}
0 & 1 & * & 0 \\
0 & 0 & 0 & 1
\end{array}\right],\left[\begin{array}{llll}
0 & 0 & 1 & 0 \\
0 & 0 & 0 & 1
\end{array}\right]} \\
& C(1,1) \quad C(1,2) \quad C(2,2)
\end{aligned}
$$

A little thought shows that $\overline{C\left(a_{1}, \cdots, a_{k}\right)} \supset C\left(b_{1}, \cdots, b_{k}\right)$ if and only if $a_{i} \leqq b_{i}$ for $1 \leqq i \leqq k$. Thus we see directly that $Q^{X} \cong L(k, n-k)$. The closure of the cell $C\left(a_{1}, \cdots, a_{k}\right)$ is called a Schubert variety, and its cohomology class is called a Schubert cycle, which we shall denote by $\Omega\left(a_{1}, \cdots, a_{k}\right)$. (A more common notation is $\Omega\left(a_{1}^{\prime}, \cdots, a_{k}^{\prime}\right)$ where $\left.a_{i}^{\prime}=n-k+i-1-a_{k-i+1}.\right)$ The Schubert cycle $\omega=$ $\Omega(0,0, \cdots, 0,1) \in H^{2}(X, \mathbb{C})$ turns out to be the class of a hyperplane section. According to a special case of Pieri's formula in the Schubert calculus, the product of $\Omega\left(a_{1}, \cdots, a_{k}\right)$ with $\omega$ in $H^{*}(X, \mathbb{C})$ is equal to the sum of all $\Omega\left(b_{1}, \cdots, b_{k}\right)$ such that $b_{i} \geqq a_{i}$ and $\sum b_{i}=1+\sum a_{i}$. In other words, $\omega \cdot \Omega\left(a_{1}, \cdots, a_{k}\right)=\sum \Omega\left(b_{1}, \cdots, b_{k}\right)$, where the sum is over all sequences $\left(b_{1}, \cdots, b_{k}\right)$ covering $\left(a_{1}, \cdots, a_{k}\right)$ in $L(k, n-k)$. Thus we 
have a direct verification of Lemma 2.2. For further information on these matters, see, for example, [26], [27], [41].

5. Type $\boldsymbol{B}_{n}$. We next turn our attention to type $B_{n}$. In this case $W$ is the group of all $n \times n$ signed permutation matrices (i.e., matrices with entries $0, \pm 1$ with one nonzero entry in every row and column). $W$ has order $2^{n} n$ ! and exponents $1,3,5, \cdots, 2 n-1$. Identify the matrix $\left(m_{i j}\right) \in W$ with the ordered pair $(\pi, \varepsilon)$, where $\pi \in \widetilde{S}_{n}$ is given by $m_{i, \pi(i)}= \pm 1$ and $\varepsilon=\left(\varepsilon_{1}, \cdots, \varepsilon_{n}\right) \in\{ \pm 1\}^{n}$ by $\varepsilon_{i}=m_{i, \pi(i)}$. We then have the multiplication rule $(\pi, \varepsilon)\left(\pi^{\prime}, \varepsilon^{\prime}\right)=\left(\pi \pi^{\prime}, \delta\right)$, where $\delta_{i}=\varepsilon_{\pi^{\prime}(i)} \varepsilon_{i}^{\prime}$. We sometimes will abbreviate a group element such as $(24513,(-1,1,-1,-1,1))$ by $\overline{2} 4 \overline{5} \overline{1} 3$, and thus regard $W$ as consisting of all "barred permutations" of $\{1,2, \cdots, n\}$. For the Coxeter generators of $W$ we take the set $S=\left\{s_{1}, \cdots, s_{n}\right\}$, where $s_{i}$ is the adjacent transposition $(i, i+1), 1 \leqq$ $i \leqq n-1$, and $s_{n}=\overline{1} 23 \cdots n$. A little thought shows that $\pi \leqq \sigma$ in $W$ if $\sigma$ can be obtained from $\pi$ by a sequence of the following seven types of operations on barred permutations:
a) $i \longrightarrow \bar{i}$,
b) $i<j \longrightarrow j>i$
c) $\bar{i}<j \longrightarrow j>\bar{i}$
d) $\bar{i}<j \longrightarrow \bar{j}>i$,
e) $\bar{i}>j \longrightarrow j<\bar{i}$
f) $i>\bar{j} \longrightarrow j<\bar{i}$
g) $\bar{i}>\bar{j} \longrightarrow \bar{j}<\bar{i}$.

For instance, Fig. 4 illustrates $W$ when $n=2$.

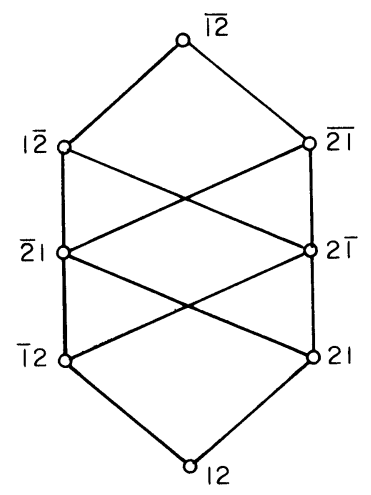

FIG. 4

If $(\pi, \varepsilon) \in W$, then one can check that

$$
\ell(\pi)=i(\pi)+\sum_{j}\left(2 d_{j}+1\right),
$$

where $i(\pi)$ is the number of inversions of $\pi, j$ ranges over all integers for which $\varepsilon_{j}=-1$, and $d_{j}$ is the number of $k$ 's appearing in $\pi=a_{1} a_{2} \cdots a_{n}$ to the left of $a_{j}$ for which $k<a_{j}$. For instance, $\ell\left(\begin{array}{lllll}\overline{3} & 1 & 5 & \overline{4} & 2\end{array}\right)=11$, since $i(\pi)=5, d_{1}=0, d_{4}=2$. It is easy to give a direct combinatorial proof that

$$
\sum_{\pi \in W} q^{\ell(\pi)}=\prod_{i=1}^{n}\left(1+q+q^{2}+\cdots+q^{2 i-1}\right),
$$

agreeing with (1). 
Now let $J \subset S$. Let $\bar{\varsigma}(a, b)$ denote the group of all signed permutations of $\{a, a+1, \cdots, b\}$. Then $W_{J}$ has the form

$$
W_{J}=\Xi\left(1, c_{1}\right) \times \Subset\left(c_{1}+1, c_{2}\right) \times \Xi\left(c_{2}+1, c_{3}\right) \times \cdots \times \Xi\left(c_{j-1}+1, n\right),
$$

where $0 \leqq c_{1}<c_{2}<\cdots<c_{j-1}<n$. The case $c_{1}=0$ corresponds to $s_{n} \notin J$. If $c_{1}=0$ then $j=n-|J| ; \quad$ otherwise $j=n-|J|+1$. Set $\quad N_{1}=\left\{1,2, \cdots, c_{1}\right\}, N_{2}=$ $\left\{c_{1}+1, \cdots, c_{2}\right\}, \cdots, N_{j}=\left\{c_{j-1}+1, \cdots, n\right\}$. One can check that $W^{J}$ consists of all $\left(a_{1} a_{2} \cdots a_{n}, \varepsilon\right) \in W$ satisfying:

(i) $\varepsilon_{i}=1$ if $a_{i} \in N_{1}$.

(ii) If $a_{r}, a_{s} \in N_{i}$ with $r<s$ and $\varepsilon_{r}=\varepsilon_{s}=1$, then $a_{r}<a_{s}$.

(iii) If $a_{r}, a_{s} \in N_{i}$ with $r<s$ and $\varepsilon_{r}=\varepsilon_{s}=-1$, then $a_{r}>a_{s}$.

(iv) If $a_{r}, a_{s} \in N_{i}$ and $\varepsilon_{r}=1, \varepsilon_{s}=-1$, then $a_{r}>a_{s}$.

For instance, if $W_{J}=\Xi(1,2) \times \Xi(3,7) \times \Xi(8,9)$, then a typical element of $W^{J}$ is $\begin{array}{lllllllll}5 & \overline{4} & 1 & \overline{8} & 6 & 2 & 7 & 9 & \overline{3}\end{array}$. The letters 1,2 are unbarred and appear in increasing order. Similarly 3, 4 are barred and decrease, 5, 6, 7 are unbarred and increase, 8 is barred and "decreases," and 9 is unbarred and "increases."

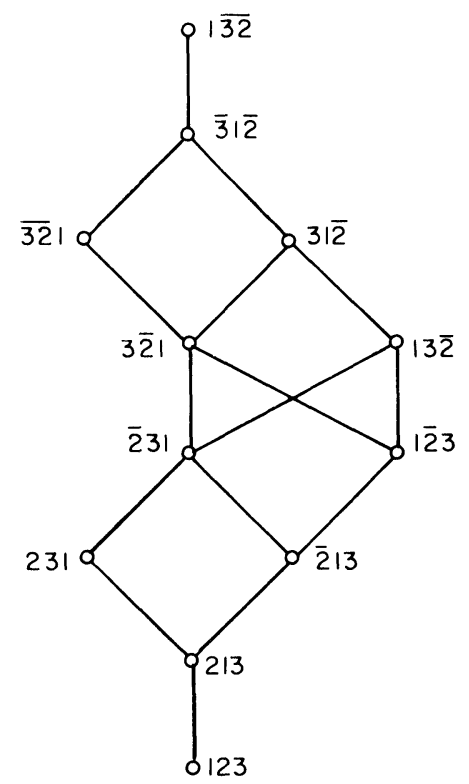

FIG. 5

Figure 5 illustrates $W^{J}$ when $n=3$ and $J=\left\{s_{1}, s_{3}\right\}$. We see that, unlike the situation for $A_{n}, W^{J}$ need not be a distributive lattice (or even just a lattice) when $J$ is a maximal subset of $S$. There is one case, however, in which $W^{J}$ is a distributive lattice, viz., $J=\left\{s_{1}, s_{2}, \cdots, s_{n-1}\right\}$, so $W_{J}=\subseteq(1, n)$. In this case we will denote $W^{J}$ by $M(n)$. To see that $M(n)$ is indeed a distributive lattice, observe that for every sequence $\varepsilon=$ $\left(\varepsilon_{1}, \cdots, \varepsilon_{n}\right) \in\{ \pm 1\}^{n}$, there is a unique $\pi \in \Im_{n}$ for which $(\pi, \varepsilon) \in M(n)$. Identify $\varepsilon$ with the subset of $\{1,2, \cdots, n\}$ consisting of those integers $t$ for which $\varepsilon_{t}=-1$. Then the partial order on $M(n)$ is given by $\left\{a_{1}, \cdots, a_{j}\right\} \leqq\left\{b_{1}, \cdots, b_{k}\right\}$ if $a_{1}<\cdots<a_{j}, b_{1}<\cdots<b_{k}$, $j \leqq k$, and $a_{j-i} \leqq b_{k-i}$ for $0 \leqq i \leqq j-1$. It is then easily seen that $M(n)$ is a distributive lattice. The poset $P$ for which $M(n)=2^{P}$ is given by $P=2^{2 \times(n-1)}$. Figure 6 illustrates $M$ (4). 


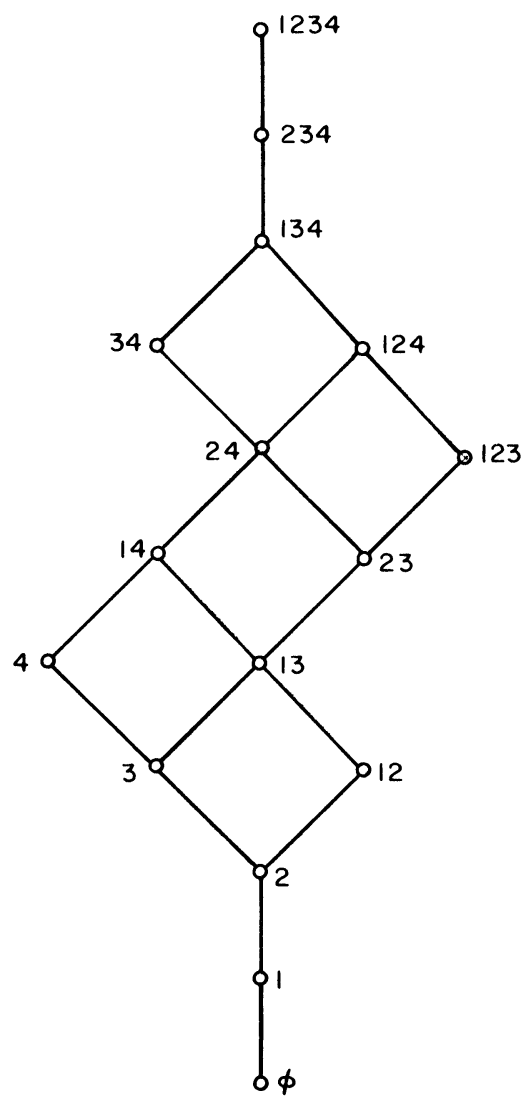

FIG. 6

Lindström [30] conjectured that $M(n)$ has property $S_{1}$, while in fact we now know that $M(n)$ has property $S$ and is rank-unimodal. (I am grateful to Larry Harper for calling my attention to Lindström's conjecture.) The rank-generating function of $M(n)$ is $(1+q)\left(1+q^{2}\right) \cdots\left(1+q^{n}\right)$. The unimodality of the coefficients of this polynomial was first explicitly proved by Hughes [25], based on a result of Dynkin (see [40] for further information). Presumably, however, this result could also be proved analytically using the methods of [12]. Lindström [30], [31] shows that the structure of $M(n)$ is related to a conjecture [13, (12)] of Erdös and Moser (see also [12], [38], [42]). In fact, Corollary 5.3 below provides a more general result. I am grateful to Ranee Gupta for pointing out an error in my original treatment of the Erdös-Moser conjecture.

Corollary 5.1. Let $A$ be a set of distinct real numbers. Assume that velements of $A$ are negative, $\zeta$ are equal to 0 (so $\zeta=0$ or 1 ), and $\pi$ are positive. Let $B_{1}, \cdots, B_{r}$ be subsets of $A$ whose element sums take on at most $k$ distinct values. Then $r$ does not exceed the sum of the $k$ middle coefficients of the polynomial

$$
G_{\nu \zeta \pi}(q)=2^{\zeta}(1+q)\left(1+q^{2}\right) \cdots\left(1+q^{\nu}\right) \cdot(1+q)\left(1+q^{2}\right) \cdots\left(1+q^{\pi}\right)
$$

(there being two equivalent choices of the " $k$ middle coefficients" when $\left(\begin{array}{c}\nu+1 \\ 2\end{array}\right)+$ $\left(\begin{array}{c}\pi+1 \\ 2\end{array}\right)-k$ is even). Moreover, this value of $r$ is achieved by taking $A=$ $\{-1,-2, \cdots,-\nu\} \cup\{1,2, \cdots, \pi\} \cup Z$, where $Z=\phi$ or $\{0\}$ depending on whether $\zeta=0$ or 1 . 
Proof. Since 0 can be adjoined to a set without affecting its element sum we may assume $\zeta=0$. Let $M(\nu)^{*}$ denote the order-dual of $M(\nu)$.(The elements of $M(\nu)$ and $M(\nu)^{*}$ coincide, but $C \leqq C^{\prime}$ in $M(\nu)^{*}$ if and only if $C \geqq C^{\prime}$ in $M(\nu)$.) Regard elements of the product $M(\nu)^{*} \times M(\pi)$ as consisting of pairs $(C, D)$, where $C$ is a subset of $\{1,2, \cdots, \nu\}$, and $D$ is a subset of $\{1,2, \cdots, \pi\}$. Suppose that the elements of $A$ are $\alpha_{\nu}<\cdots<\alpha_{1}<0<\beta_{1}<\cdots<\beta_{\pi}$ and that $B_{s}=\left\{\alpha_{i_{1}}, \cdots, \alpha_{i_{h}}, \beta_{j_{1}}, \cdots, \beta_{j_{m}}\right\}$. Associate with $B_{s}$ the set $\left(C_{s}, D_{s}\right)=\left(\left\{i_{1}, \cdots, i_{h}\right\},\left\{j_{1}, \cdots, j_{m}\right\}\right) \in M(\nu)^{*} \times M(\pi)$. It is easy to see that the subset $\left\{\left(C_{1}, D_{1}\right), \cdots,\left(C_{r}, D_{r}\right)\right\}$ of $M(\nu)^{*} \times M(\pi)$ contains no $(k+1)$-element chain provided there are most $k$ distinct element sums of $B_{1}, \cdots, B_{r}$. Now it is not difficult to see that $M(\nu)^{*} \cong M(\nu)$. (For instance, given the set $T=\left\{i_{1}, \cdots, i_{h}\right\} \in M(\nu)$ with $1 \leqq i_{1}<\cdots<i_{h} \leqq \nu$, define $T^{*}$ to be the set of nonzero parts of the partition $\lambda$ which is conjugate (in the sense of $[9$, p. 100]) to the partition whose parts are $\nu-i_{h}, \nu-1-i_{h-1}, \cdots, \nu-h+1-i_{1}, \nu-h, \nu-h-1, \cdots, 1$. Then the mapping $T \rightarrow T^{*}$ is an isomorphism $M(\nu) \rightarrow M(\nu)^{*}$. See also $\S 7$ for a more general result.) The proof now follows from Theorem 3.1 and Proposition 2.5 (or from Theorem 3.1 alone applied to the appropriate reducible Weyl group) and the fact that the rank-generating function of $M(\nu)^{*} \times M(\pi)$ is $G_{\nu 0 \pi}(q)$.

We now want to consider the situation where $\nu+\zeta+\pi$ is fixed, but $\nu, \zeta$, and $\pi$ can vary. First we need:

LEMMA 5.2. Let $G(q)$ be a polynomial of degree $d$ with symmetric unimodal coefficients. Fix positive integers $j$ and $k$. Then the sum of the middle $k$ coefficients of $G(q)\left(1+q^{j+1}\right)$ does not exceed the sum of the middle $k$ coefficients of $G(q)\left(1+q^{j}\right)$.

Proof. Let $G(q)=\alpha(0)+\alpha(1) q+\cdots+\alpha(d) q^{d}$. For simplicity of notation we assume $d=2 d^{\prime}, j=2 j^{\prime}, k=2 k^{\prime}$. The other cases are done similarly. The middle $k$ coefficients of $G(q)\left(1+q^{j}\right)$ are

$$
\alpha\left(d^{\prime}+j^{\prime}-k^{\prime}+i\right)+\alpha\left(d^{\prime}-j^{\prime}-k^{\prime}+i\right), \quad 0 \leqq i \leqq k-1 .
$$

The middle $k$ coefficients of $G(q)\left(1+q^{j+1}\right)$ are

$$
\alpha\left(d^{\prime}+j^{\prime}-k^{\prime}+i+1\right)+\alpha\left(d^{\prime}-j^{\prime}-k^{\prime}+i\right), \quad 0 \leqq i \leqq k-1 .
$$

(Here we set $\alpha(t)=0$ if $t<0$.) If $\Omega$ applied to a polynomial denotes the sum of its middle $k$ coefficients, then

$$
\Omega G(q)\left(1+q^{j}\right)-\Omega G(q)\left(1+q^{j+1}\right)=\alpha\left(d^{\prime}+j^{\prime}-k^{\prime}\right)-\alpha\left(d^{\prime}+j^{\prime}+k^{\prime}\right) .
$$

Since $\alpha(i)=\alpha(d-i)$ and $\alpha(0) \leqq \alpha(1) \leqq \cdots \leqq \alpha\left(d^{\prime}\right)$, it follows that $\alpha\left(d^{\prime}+j^{\prime}-k^{\prime}\right) \geqq$ $\alpha\left(d^{\prime}+j^{\prime}+k^{\prime}\right)$, completing the proof.

COROllary 5.3. Let $A$ be a set of $n$ distinct real numbers, and let $B_{1}, \cdots, B_{r}$ be subsets of $A$ whose element sums take on at most $k$ distinct values. Let $\nu=[(n-1) / 2]$ and $\pi=[n / 2]$. Then $r$ does not exceed the sum of the $k$ middle coefficients of the polynomial

$$
2(1+q)\left(1+q^{2}\right) \cdots\left(1+q^{\nu}\right) \cdot(1+q)\left(1+q^{2}\right) \cdots\left(1+q^{\pi}\right) .
$$

Moreover, this value of $r$ is achieved by choosing $A=\{-\nu,-\nu+1, \cdots, \pi\}$.

Proof. For fixed $n=\nu+\zeta+\pi$, it follows from Lemma 5.2 that the sum of the middle $k$ coefficients of $G_{\nu \zeta \pi}(q)$ is maximized by choosing $\zeta=1, \nu=[(n-1) / 2], \pi=[n / 2]$. The proof follows from Corollary 5.1.

The actual conjecture [13,(12)] of Erdös and Moser is equivalent to the case $k=1$, and $n$ odd, of Corollary 5.3. A purely combinatorial derivation of the Erdös-Moser conjecture from the fact that $M(n)$ has property S appears in [35]. 
6. Type $D_{n}$. If $(W, S)$ is a Coxeter system of type $D_{n}$, then $W$ is the subgroup of the group $W^{\prime}$ of type $B_{n}$ consisting of all $(\pi, \varepsilon)$ such that $\prod_{i=1}^{n} \varepsilon_{i}=+1$. W has order $2^{n-1} n$ ! and exponents $1,3,5, \cdots, 2 n-5,2 n-3, n-1$. We may take $S=\left\{s_{1}, \cdots, s_{n}\right\}$ where $s_{i}=(i, i+1)$ if $1 \leqq i \leqq n-1$ (as in type $\left.B_{n}\right)$ and $s_{n}=\overline{2} \overline{1} 34 \cdots n$. We then have the following seven transformation rules for obtaining $w^{\prime}$ from $w$ when $w \leqq w^{\prime}$ in $W$ :
a) $i<j \longrightarrow \bar{j}>\bar{i}$,
b) $i<j \longrightarrow j>i$,
c) $i<j \longrightarrow j>\bar{i}$
d) $\bar{i}<j \longrightarrow \bar{j}>i$,
e) $\bar{i}>j \longrightarrow j<\bar{i}$
f) $i>\bar{j} \longrightarrow j<\bar{i}$,
g) $\bar{i}>\bar{j} \longrightarrow \bar{j}<\bar{i}$.

Note that rules b-g coincide with those for $B_{n}$, and that rule a for $D_{n}$ is obtained by applying rule $\mathrm{b}$ and rule a twice for $B_{n}$. It follows that if $\pi \leqq \sigma$ in $W$ then $\pi \leqq \sigma$ in $W^{\prime}$. The converse, however, is false. For instance, $21<\overline{21}$ in $W^{\prime}$ but 21 and $\overline{21}$ are incomparable in $W$. Figure 7 depicts $W$ when $n=2$.

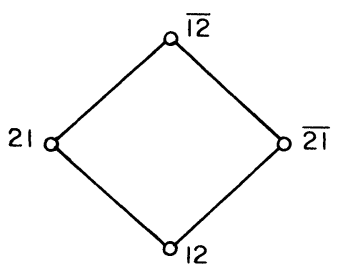

FIG. 7

If $(\pi, \varepsilon) \in W$, then

$$
\ell(\pi)=i(\pi)+2 \sum_{j} d_{j},
$$

where $i(\pi)$ and $d_{j}$ have the same meaning as in (6). For instance, $\ell\left(\begin{array}{lllll}\overline{3} & 1 & 5 & \overline{4} & 2\end{array}\right)=9$ for $D_{5}$, while $\ell\left(\begin{array}{lllll}\overline{3} & 1 & 5 & \overline{4} & 2\end{array}\right)=11$ for $B_{5}$.

Now let $J \subset S$. In so far as describing the poset $W^{J}$ is concerned, we may assume that if $s_{n}=\overline{2134} \cdots n \in J$ then also $s_{1}=213 \cdots n \in J$, since interchanging $s_{1}$ and $s_{n}$ induces an automorphism of the Coxeter system $(W, S)$. Thus if we let $\overleftarrow{\Xi}(a, b)$ denote the group of all signed permutations of $\{a, a+1, \cdots, b\}$ with an even number of -1 's, then $W_{J}$ has the form

$$
W_{J}=\Im\left(1, c_{1}\right) \times \Im\left(c_{1}+1, c_{2}\right) \times \cdots \times \Im\left(c_{j-1}+1, n\right),
$$

where $0 \leqq c_{1}<c_{2}<\cdots<c_{j-1}<n$ and $c_{1} \neq 1$. The case $c_{1}=0$ corresponds to $s_{n} \notin J$. Defining $N_{1}=\left\{1,2, \cdots, c_{1}\right\}, N_{2}=\left\{c_{1}+1, \cdots, c_{2}\right\}, \cdots, N_{i}=\left\{c_{i-1}+1, \cdots, n\right\}$, one can check that $W^{J}$ consists of all $\left(a_{1} a_{2} \cdots a_{n}, \varepsilon\right) \in W$ satisfying:

(i) $\varepsilon_{1}=1$ if $a_{i} \in N_{1}$ and $a_{i}>1$.

(ii)-(iv) Same as for type $B_{n}$.

(v) 1 precedes every other element of $N_{1}$ (even if 1 is barred).

For instance, Fig. 8 depicts $W^{J}$ when $n=3$ and $J=\{12\}$, i.e., $W_{J}=$ $\tilde{S}(1,2) \times \Im(3,3)$, so $N_{1}=\phi, N_{2}=\{1,2\}, N_{3}=\{3\}$. Note that this poset is isomorphic to that of Fig. 2 ; this is no accident since Coxeter systems of types $A_{3}$ and $D_{3}$ are isomorphic. (Recall that to obtain nonisomorphic systems, one may take $A_{n}$ for $n \geqq 1$, $B_{n}$ for $n \geqq 2$, and $D_{n}$ for $n \geqq 4$.) 


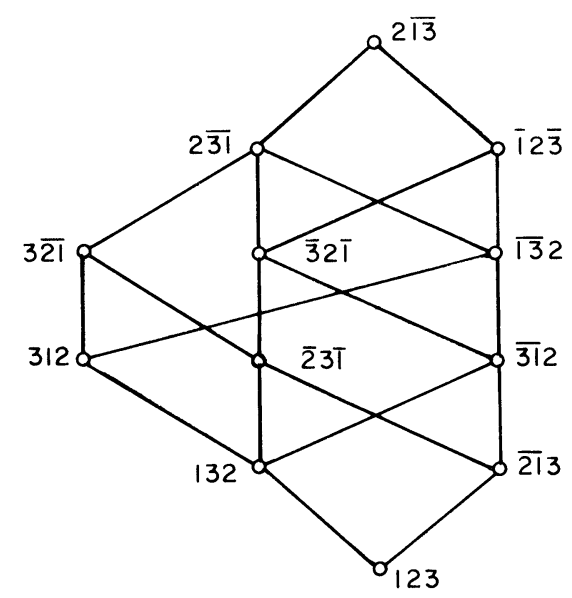

FIG. 8

As in the case of $B_{n}, W^{J}$ need not be a distributive lattice when $J$ is maximal. For instance, take $n=4$ and $J=\left\{s_{1}, s_{3}, s_{4}\right\}=S-\{(23)\}$, so $W_{J}=\widehat{\Xi}(1,2) \times \Im(3,4)$. Then the rank-generating function of $W^{J}$ is given by

$$
F\left(W^{J}, q\right)=1+q+3 q^{2}+3 q^{3}+4 q^{4}+4 q^{5}+3 q^{6}+3 q^{7}+q^{8}+q^{9},
$$

and it is easy to check that there does not exist a distributive lattice with this rank-generating function. As in the situation for $B_{n}$, there is one special case for which $W^{J}$ is a distributive lattice. Take $J=\left\{s_{1}, s_{2}, \cdots, s_{n-1}\right\}$, so $W_{J}=\subseteq(1, n)$. If we regard $M(n)$ (as defined in the previous section) as consisting of all subsets of $\{1,2, \cdots, n\}$, then $W^{J}$ turns out to be the subposet of $M(n)$ consisting of all sets of even cardinality. But it is easily seen that this subposet is isomorphic to $M(n-1)$, so nothing new is obtained.

7. Final comments. In view of the examples $L(m, n)$ and $M(n)$, it is natural to ask under what circumstances is $W^{J}$ a distributive lattice. I am grateful to Robert Proctor for supplying the following answer to this question. The Coxeter generators $S$ of an irreducible Weyl group $W$ correspond to the fundamental representations $\lambda_{i}(1 \leqq i \leqq n)$ of a certain complex simple Lie algebra $g$. By direct computation facilitated by representation theory, Proctor has shown that (except for the representations $\lambda_{1}$ and $\lambda_{2}$ of $G_{2}$ ) $W^{J}$ is distributive if and only if the irreducible representation of $g$ with highest weight $\sum_{i \notin J} \lambda_{i}$ is miniscule, as defined in [6, p. 226]. These representations have special significance in other contexts; see [39] and more generally [28]. It turns out that for all the distributive $W^{J}$ 's except $L(m, n)$ and $M(n)$, it is easy to check Property $S$ directly.

Proctor has also shown that if $W$ is a Weyl group with largest element $v$ (in the Bruhat order) and if $W^{J}$ (for any $J \subset S$ ) has largest element $y$, then the bijection from $W^{J}$ to $W^{J}$ given by $w \rightarrow v w y^{-1} v^{-1}$ is an anti-automorphism of $W^{J}$. Thus $W^{J}$ is self-dual whenever $W$ is a Weyl group. We do not know whether the more general posets $Q^{X}$ of Theorem 2.4 need always be self-dual.

We conclude with an open problem. Let $P$ be a finite graded rank-symmetric poset of rank $n$, with rank function $\rho$. $P$ is called a symmetric chain order (e.g., [17, §3], [20], [21]) if it can be partitioned into pairwise disjoint saturated chains $x_{i}<x_{i+1}<\cdots<x_{n-i}$ such that $\rho\left(x_{j}\right)=j$. It is easy to see that a symmetric chain order satisfies Property $T$ and hence is rank-unimodal. Easy examples show that a rank-symmetric poset satisfying Property $T$ need not be a symmetric chain order. 
Our open problem is the following: Are all the posets $Q^{X}$ of Theorem 2.4 (or at least the special cases $W^{J}$ of Theorem 3.1) symmetric chain orders? Since any poset $Q^{X}$ given by Theorem 2.4 has property $T$, there are pairwise disjoint chains connecting all of $Q_{i}^{X}$ to $Q_{i+1}^{X}$ when $i<n / 2$, and all of $Q_{i}^{X}$ to $Q_{i-1}^{X}$ when $i>n / 2$. Piecing together these chains yields a partition of $Q^{X}$ into saturated chains all of which pass through the middle rank (when $n$ is even) or middle two ranks (when $n$ is odd). However, it is by no means clear whether these chains may be chosen to be symmetric about the middle.

Emden Gansner has pointed out to me that for type $A_{n}$, there is a rank-preserving, order-preserving bijection $\mathbf{1} \times \mathbf{2} \times \cdots \times \mathbf{n} \stackrel{\varphi}{\rightarrow} W=\mathfrak{\Im}_{n}$, where $\mathbf{1} \times \mathbf{2} \times \cdots \times \mathbf{n}=$ $\left\{\left(b_{1}, \cdots, b_{n}\right): 0 \leqq b_{i}<i\right\}$. Namely, $\varphi\left(b_{1}, \cdots, b_{n}\right)$ is that permutation $\pi=a_{1} a_{2} \cdots a_{n} \in$ $\widetilde{S}_{n}$ such that $b_{i}$ is the number of elements $j$ appearing in $\pi$ to the right of $i$ and satisfying $j<i$. Since any product of chains is a symmetric chain order (e.g., [17, pp. 30-31]), it follows that $\widetilde{S}_{n}$ (with the Bruhat order) is also a symmetric chain order. A similar argument for types $B_{n}$ and $D_{n}$ produces rank-preserving order-preserving bijections $\mathbf{2} \times \mathbf{4} \times \cdots \times \mathbf{2 n} \rightarrow \widetilde{\Phi}_{n}$ and $\mathbf{2} \times \mathbf{4} \times \cdots \times \mathbf{2}(\mathbf{n}-\mathbf{1}) \times \mathbf{n} \rightarrow \widehat{\Xi}_{n}$. Hence $\overline{\mathfrak{S}}_{n}$ and $\widetilde{S}_{n}$ are also symmetric chain orders. However, we do not know for instance whether $L(m, n)$ and $M(n)$ are always symmetric chain orders. Lindström [32] has shown that $L(3, n)$ is a symmetric chain order, and D. West $[44]$ has shown that $L(4, n)$ is a symmetric chain order. Littlewood [33, pp. 193-203] claims to prove that $L(m, n)$ is indeed a symmetric chain order for all $m$ and $n$. However, his proof is invalid. Specifically, it relies on the "method of chains" of Aitken [45], and this method is not correct as stated by Aitken. For the reader's benefit we will discuss the nature of Aitken's error in more detail. Let $P=\left\{x_{1}, \cdots, x_{n}\right\}$ be a finite poset, and let $\Phi=\left(a_{i j}\right)$ be the $n \times n$ matrix defined by $a_{i j}=0$ unless $x_{i}<x_{j}$ in $P$; otherwise the $a_{i j}$ 's are independent indeterminates over $\mathbb{Q}$. Remove a chain $C_{1}$ of maximum cardinality $c_{1}$ from $P$, then remove a chain $C_{2}$ of maximum cardinality $c_{2}$ from $P-C_{1}$, etc. Aitken essentially claims first that the numbers $c_{1}, c_{2}, \cdots$, are independent of the choice of chains $C_{1}, C_{2}, \cdots$, and second that the numbers $c_{1}, c_{2}, \cdots$ are the sizes of the Jordan blocks of $\Phi$. The first claim is clearly false. However, Littlewood's proof would still be valid if there were some way of choosing $C_{1}, C_{2}, \cdots$ so that the second claim is true. Even this weaker result is false. Let $P$ be the poset of Fig. 9. We have no choice but to take $c_{1}=4, c_{2}=1, c_{3}=1$. However, the Jordan block sizes of $\Phi$ are 4 and 2. A corrected version of Aitken's result appears in [37]. If this corrected result is used in conjunction with Littlewood's method, it yields the result that $L(m, n)$ has property $\mathrm{T}$. Thus we have an alternative proof, avoiding the hard Lefschetz theorem (though actually Littlewood's method essentially proves the hard Lefschetz theorem for the Grassmann variety), that $L(m, n)$ has property $\mathrm{T}$.

A further property of posets which implies the Sperner property is the LYM property $[17, \S 4]$. However, Griggs has observed that $L(4,3)$ fails to satisfy the LYM property.

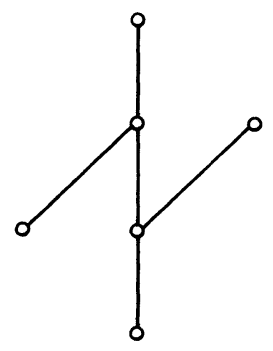

FIG. 9 
Note added in proof. A proof that $L(3, m)$ and $L(4, m)$ have symmetric chain decompositions was first given by W. Riess, Zwei Optimierungsprobleme auf Ordnungen, Arbeitsberichte des Institute für Mathematische Maschinen und Datenverarbeitung (Informatik) 11, Number 5, Erlangen, April 1978.

\section{REFERENCES}

[1] A. C. Aitken, Determinants and Matrices, 3rd ed., Oliver and Boyd, London, 1944.

[2] G. E. ANDREWs, $\boldsymbol{A}$ theorem on reciprocal polynomials with applications to permutations and compositions, Amer. Math. Monthly, 82 (1975), pp. 830-833.

[3] G. BIRKhoff, Lattice Theory, third ed., American Mathematical Society, Providence, RI, 1967.

[4] A. Borel AND A. HAEFliger, La classe d'homologie fondamentale d'un espace analytique, Bull. Soc. Math. France, 89 (1961), pp. 461-513.

[5] A. Borel and J. Tits, Compléments à l'article: "Groupes réductifs," Inst. Hautes Études Sci. Publ. Math., No. 41 (1972), pp. 253-276.

[6] N. BouRBAKI, Groupes et algèbres de Lie, Éléments de Mathématique, Fasc. XXXIV, Hermann, Paris, 1968.

[7] E. R. CANFIELD, A Sperner property preserved by product, Linear and Multilinear Algebra, to appear.

[8] R. W. CARTER, Simple Groups of Lie Type, Wiley, New York, 1972.

[9] L. COMTET, Advanced Combinatorics, Reidel, Boston, 1974.

[10] M. CORNAlba AND P. A. GRIFFITHS, Some transcendental aspects of algebraic geometry, Algebraic Geometry, Arcata 1974, R. Hartshorne, ed., American Mathematical Society, Providence, RI, 1975, pp. 3-110.

[11] V. V. DEODHAR, Some characterizations of Bruhat ordering on a Coxeter group and determination of the relative Möbius function, Invent. Math., 39 (1977), pp. 187-198.

[12] R. C. ENTRINGeR, Representation of $m$ as $\sum_{k=-n}^{n} \varepsilon_{k} k$, Canad. Math. Bull., 11 (1968), pp. 289-293.

[13] P. ERDös, Extremal problems in number theory, Theory of Numbers, A. L. Whiteman, ed., American Mathematical Society, Providence, RI, 1965, pp. 181-189.

[14] W. Fulton, Rational equivalence on singular varieties, Inst. Hautes Etudes Sci. Publ. Math., no. 45 (1975), pp. 147-167.

[15] C. GREENE, Some partitions associated with a partially ordered set, J. Combinatorial Theory, 20 (1976), pp. 69-79.

[16] C. Greene And D. J. Kleitman, The structure of Sperner $k$-families, J. Combinatorial Theory, 20 (1976), pp. 41-68.

[17] C. Greene AND D. J. Kleitman, Proof techniques in the theory of finite sets, Studies in Combinatorics, G.-C. Rota, ed., Mathematical Association of America, Washington, DC, 1978, pp. 22-79.

[18] P. GRIFFITHS AND J. ADAMS, Topics in Algebraic and Analytic Geometry, Princeton University Press, Princeton, NJ, 1974.

[19] P. Griffiths AND J. HARris, Principles of Algebraic Geometry, Wiley, New York, 1978.

[20] J. R.: GRIGGS, Sufficient conditions for a symmetric chain order, SIAM J. Appl. Math., 32 (1977), pp. 807-809.

[21] J. R. GRIGGS, On chains and Sperner $k$-families in ranked posets, J. Combinatorial Theory, to appear.

[22] A. GROTHENDIECK, Sur quelques propriétés fondamentales en théorie des intersections, Séminaire C. Chevalley, Ecole Normale Supérieure, Paris, 2 (1958), Chap. 4.

[23] L. H. HARPER, Stabilization and the edgesum problem, Ars Combinatoria, 4 (1977), pp. 225-270.

[24] W. V. D. HodGe, The Theory and Applications of Harmonic Integrals, 2nd ed., Cambridge University Press, London, 1952.

[25] J. W. B. HuGHES, Lie algebraic proofs of some theorems on partitions, Number Theory and Algebra, H. Zassenhaus, ed., Academic Press, New York, 1977, pp. 135-155.

[26] S. L. Kleiman, Problem 15. Rigorous foundation of Schubert's enumerative calculus, Mathematical Developments Arising from Hilbert Problems, F. E. Browder, ed., American Mathematical Society, Providence, RI, 1976, pp. 445-482.

[27] S. L. KLeiman AND D. LaKsov, Schubert calculus, Amer. Math. Monthly, 79 (1972), pp. 1061-1082.

[28] V. Lakshmibai, C. Musili And C. S. Seshadri, Geometry of $G / P$, Bull. Amer. Math. Soc., 1 (1979), pp. 432-435.

[29] S. LeFsCHETZ, L'Analysis situs et la Géométrie Algébrique, Gauthier-Villars, Paris, 1924; reprinted in Selected Papers, Chelsea, New York, 1971. 
[30] B. Lindoström, Conjecture on a theorem similar to Sperner's, Combinatorial Structures and their Applications, R. Guy, H. Hanani, N. Sauer, and J. Schonheim, eds., Gordon and Breach, New York, 1970, p. 241.

[31] B. LiNDSTRÖM, Om en elementär sats i kombinatoriken och nágra tillämpningar, Nordisk Mat. Tidskr., 17 (1969), pp. 61-70.

[32] - A partition of $L(3, n)$ into saturated symmetric chains, European J. Combinatorics, to appear.

[33] D. E. LitTlewood, The Theory of Group Characters and Matrix Representations of Groups, 2nd ed., Oxford University Press, London, 1950.

[34] W. Messing, Short sketch of Deligne's proof of the hard Lefschetz theorem, Algebraic Geometry, Arcata 1974, R. Hartshorne, ed., American Mathematical Society, Providence, RI, 1975, pp. 563-580.

[35] G. W. PECK, Erdös' conjecture on sums of distinct numbers, Studies in Applied Math., to appear.

[36] R. A. Proctor, M. E. Saks And D. G. Sturtevant, Product partial orders with the Sperner property, Discrete Math., to appear.

[37] M. E. SAKs, Dilworth numbers, incidence maps and product partial orders, this Journal, this issue pp. 211-215.

[38] A. SÁRKözI AND E. SzemerÉdi, Über ein Problem von Erdös und Moser, Acta Arith., 11 (1966), pp. 205-208.

[39] C. S. SESHADRI. Geometry of G/P-I (Standard monomial theory for a miniscule P), C. P. Ramanujan: A Tribute, Springer-Verlag, 1978, published for the Tata Institute of Fundamental Research, Bombay.

[40] R. STANLEY, Unimodal sequences arising from Lie algebras, Proc. Young Day, to appear.

[41] - Some combinatorial aspects of the Schubert calculus, Combinatoire et Représentation du Groupe Symétrique, D. Foata, ed., Lecture Notes in Math. No. 579, Springer-Verlag, New York, 1977, pp. 217-251.

[42] J. H. VAN LiNT, Representation of $O$ as $\sum_{k=-N}^{N} \varepsilon_{k} k$, Proc. Amer. Math. Soc., 19 (1967), pp. 182-184.

[43] A. WeIL, Introduction à l'étude des variétés Kähleriennes, Hermann, Paris, 1958.

[44] D. B. WEST, A symmetric chain decomposition of $L(4, n)$, preprint.

[45] A. C. Aitken, The normal form of compound and induced matrices, Proc. London Math. Soc., (2) 38 (1934), pp. 354-376. 